\title{
Codependence in the Nephromyces species swarm depends on heterospecific bacterial endosymbionts
}

\author{
Christopher Paight, Elizabeth Sage Hunter, Christopher E Lane
}

02881, U.S.A.

Department of Biological Sciences, University of Rhode Island, Kingston, RI,

\begin{abstract}
The phylum Apicomplexa encompasses 6000 ubiquitous animal parasites, including Plasmodium, the most deadly human parasite on Earth. Anciently parasitic lineages, like apicomplexans, lose core metabolic pathways over time, as they evolve less costly scavenging mechanisms. The recent description of a mutualistic apicomplexan, Nephromyces, from deep within this parasitic group, opened the possibility of an evolutionary innovation that allowed an escape from a parasitic lifestyle. Nuclear genome data from Nephromyces, as well as the three bacterial symbionts that live within this species complex, demonstrate that the bacteria within Nephromyces contribute essential cofactors and amino acids that have enabled Nephromyces to abandon a parasitic lifestyle. Among these, bacterial lipoic acid appears to be a key cofactor for the reduction of virulence in Nephromyces. However, whereas we use FISH microscopy to reveal that each individual Nephromyces harbors no more than one endosymbiont type, no single bacterial endosymbiont can account for all missing metabolites. Based on the unique habitat of Nephromyces, as well as genomic, culturing, and wild population data, we conclude that Nephromyces has evolved as an extraordinary clade of codependent species, unlike any previously described.
\end{abstract}

\section{Introduction}

Symbiosis is one of the most important evolutionary processes shaping biodiversity on Earth. Symbiotic communities often bring together organisms from different domains of life, which can provide an unparalleled route to evolutionary innovation, allowing life to thrive in unconventional niches such as hydrothermal vents (1) or the oxic-anoxic interface (2). One such extreme environment is the renal sac of the marine invertebrate genus Molgula. These tunicates produce a liquid-filled organ, which contains high enough concentrations of uric and oxalic acids to form crystal structures analogous to human kidney stones (3). Surprisingly, within this environment live multiple apicomplexan species of Nephromyces (Fig. 1 ), with vertically transmitted bacterial endosymbionts living inside them (4). Unlike the $\sim 6000$ described species of apicomplexans, Nephromyces spp. are not parasitic (5). Instead, they maintain a mutualistic or commensal relationship with molgulid tunicates, using a combination of retained metabolic pathways and their bacterial endosymbionts (6). 
Besides Nephromyces, the phylum Apicomplexa is composed entirely of obligate metazoan parasites. As a result of an estimated 800 million years of evolution as obligate parasites, many of the genomic patterns associated with the response to a parasitic lifestyle have been described from the genomes of apicomplexan species (7). Apicomplexan-specific examples include expansions in the Plasmodium var protein family, which are involved in host manipulation and evasion, as well as an expansion of rhoptry, microneme, and dense granule proteins $(8,9)$. The list of core biosynthetic pathways apicomplexans have lost includes purine biosynthesis, purine degradation, biosynthesis of many amino acids, and vitamin biosynthesis (10-13). These losses make the parasite dependent on the host, not only for primary carbon and nitrogen, but also for any metabolites it can no longer generate, either by de novo synthesis or conversion. High demand on the host for these metabolites to fuel parasite growth increases the cost of infection, thereby increasing virulence. Parasites must maintain a delicate balance between transmission, virulence, and host immune system evasion.

The trade-offs in this balance have been described in detail (14-16), but one common solution many parasites adopt is maintaining low relative abundance inside the host. Higher parasite abundance will increase the cost to that host, and increase virulence. If the parasites kill the host before completing their lifecycle or before transmission to a new host, their fitness falls to zero. Similarly, if parasites have a high prevalence in a population and high lethality, they risk decimating their host population. High-sustained infection prevalence is a good indicator of low virulence (17), and low virulence is often achieved by self-limited reproduction by the parasites. In this way, Nephromyces stands out as a very atypical parasite.

Nephromyces has a nearly $100 \%$ infection rate, which is sustained almost year-round (5). Based on typical host/parasite dynamics, Nephromyces also reaches unexpectedly high cell densities. These atypical epidemiological factors were the basis for the conclusion that Nephromyces must be mutualistic (5). In order to reach high cell densities while maintaining low virulence, Nephromyces was predicted to produce something of high value to the host, to offset the cost associated with maintaining such high densities of an obligate parasite. Nephromyces is also unusual because it lives the majority of its life extracellularly (18). Whereas some early-diverging gregarines are primarily extracellular, the Nephromycidae resolves as sister to the Hematozoans, a clade of obligate intracellular blood parasites (19). While it remains unknown if Nephromyces is capable of forming direct connections to tunicate host cells, there is no microscopic support for direct cell-to-cell connections. Instead, Nephromyces cells are found independent of tunicate cells in the lumen of the renal sac. The renal sac is a large ductless structure of unknown function exclusively found in a Molgulidae family of tunicates (20).

Despite its name, the renal sac does not function as a typical renal organ but was named for the large deposits of crystallized uric acid and calcium oxalate. Many ascidians have localized deposits of uric acid, but tunicates in the Molgulidae family 
have the largest uric acid deposits (21). While the function of these deposits of uric acid in the tunicate remain unclear, previous work demonstrated that Nephromyces is able to degrade uric acid, because it retains the ancestral purine degradation genes lost in all other apicomplexans (6). Based on transcriptome data and pathway analysis, uric acid may be the primary source of carbon and nitrogen for Nephromyces (6). Uric acid is an atypical source of carbon and nitrogen, but not completely unknown. There are several species of bacteria and fungi that can be cultured on media only uric acid (22-24).

The shift from an intracellular environment rich in preformed metabolites and precursor molecules, to an extracellular lifestyle inside the renal sac, likely limited the amount and type of preformed metabolites available for the ancestor of Nephromyces to scavenge. Given the large number of basic biosynthetic pathways lost in apicomplexans, this organism would have needed to evolve mechanisms of obtaining these essential metabolites not encoded in its genome. Metabolites could still be scavenged from the host; while not as abundant, many essential metabolites are still present in the extracellular environment (25). Another potential source of these metabolites could be the development of de novo synthesis pathways. However, the sheer number of essential pathways missing in apicomplexans makes the transition to extracellular unlikely. A third option for filling the need for so many basic metabolites is the acquisition of bacterial endosymbionts.

Preliminary fluorescence in situ hybridization microscopy performed on Nephromyces indicated species harbor three bacterial endosymbionts: an alphaproteobacteria endosymbiont ( $\mathrm{N} \alpha$ e), Betaproteobacteria $(\mathrm{N} \beta$ e), and a Bacteroidetes (Nbe). Acquisition and maintenance of bacterial endosymbionts is a common way for eukaryotes to gain new metabolic pathways and capabilities. The functional capabilities of bacterial endosymbionts exploited by eukaryotic hosts include amino acid metabolism and vitamin metabolism (26), nitrogen metabolism (27), defense (28), chemotrophic energy production (29), and photosynthesis (30), to name a few. Whereas bacterial endosymbionts are common in many protist lineages, they are unknown in the phylum Apicomplexa outside the Nephromycidae. Previous speculation (5) that Nephromyces bacterial endosymbionts are responsible for the high levels of purine degradation observed have recently been rejected (6). However, the bacterial endosymbionts were likely instrumental in the transition from intracellular to extracellular and colonization of the renal sac.

Here we show that the diversity of Nephromyces spp. and their endosymbionts are unexpectedly high within each renal sac and forms a codependent community within their host. We determined that as many as 20 different species of Nephromyces inhabit a typical renal sac. Whereas Nephromyces species can host three different types of bacterial endosymbionts, FISH microscopy allowed us to show that only a single bacterial type exists within any one individual. Through the reconstruction and analyses of the endosymbiont bacterial genomes, we infer that each type supplies its Nephromyces host with different metabolites. 
Given that no individual species of Nephromyces, in combination with its endosymbiont, can produce a complete set of essential amino acids, we hypothesize that each of them depends on multiple congeners to meet its needs. Growth experiments demonstrate that individual Nephromyces species cannot form a viable infection. Nephromyces has thus evolved as an extraordinary clade of codependent species.

\section{Results}

\section{FISH Microscopy}

Fluorescence in situ hybridization (FisH) of wild Molgula manhattensis tunicates revealed persistent co-infections of alphaproteobacteria and Bacteroides. While these two classes of bacterial endosymbionts were frequently found together in the same renal sac, they never co-occurred in the same Nephromyces cell. A betaproteobacteria probe was also used in all experiments, but was never visualized in our samples, in contrast to (31). Based on the corresponding amplicon data for tunicates, we believe this was a problem with probe specificity and binding, and not a genuine absence of betaproteobacteria endosymbionts. All three probes have successfully hybridized in previous experiments (31), and no individual Nephromyces cells were found to contain more than one class of endosymbiont.

\section{$\underline{\text { RNA Sequencing Results }}$}

High-quality RNA was extracted from the contents of a single renal sac, resulting in 195,694 transcripts from Molgula manhattensis, Nephromyces, and the bacterial endosymbionts. After binning by species, 60,223 transcripts were attributed to Nephromyces, and 6,589 were attributed to the bacterial endosymbionts. The large number of transcripts attributed to Nephromyces was due to multiple species infecting a single host (estimated 69\% duplication). Clustering by percent sequence identity resulted in 26,938 at $90 \%, 23,850$ at $80 \%, 21,762$ at $70 \%, 19,540$ at $60 \%, 16,668$ at $50 \%$. Due to the multi-species community, the transcriptome is from several species of Nephromyces, but we estimate that there are between 8,000 and 12,000 unique transcripts in Nephromyces. KEGG functionally predicts 6,987 . BUSCO was used to assess the completeness of Nephromyces transcriptome resulting in $81.8 \%$ complete transcripts and $6.3 \%$ partial.

\section{Nephromyces Genome}

Due to the multispecies nature of the system, the genome and transcriptome for Nephromyces are pan-genomic and pan-transcriptomic, and we cannot rule out that some of our assembled contigs and transcripts are potentially chimeric. However, these limitations do not limit our characterization of the metabolic contribution of Nephromyces as a genus. The Nephromyces pan-genome assembled into 2,156 contigs has an N50 50,747 bp with 36\% GC content, a sum length of 
$60,134,441 \mathrm{bp}$, and BUSCO estimated completeness of $51.1 \%$ and duplication is $13.5 \%$.

There are 3,070 predicted protein-coding genes in the Nephromyces dataset. Of these protein-coding genes, 2,834 were recovered in the transcriptome, 236 are unique to the genome, and 794 protein-coding genes in the transcriptome are missing from the genome. The incompleteness of the genome makes determining the true absence of genes impossible, but by combining it with the more complete transcriptome, we are able to better predict the metabolic capabilities and contributions of Nephromyces to the system. The genome also provides support that transcripts binned as Nephromyces based on phylogeny were binned correctly.

\section{Endosymbiont Genome}

The genomes of three bacterial endosymbionts were retrieved from the metagenome of Nephromyces. These genomes were small, gene dense, and AT-rich . The presence of two closely related $\alpha$-proteobacteria with different genome organization limited our ability to assemble the genome onto a single contig. The $\alpha$-proteobacteria bacteria assembled into 11 contigs ranging from $13 \mathrm{~kb}$ to $312 \mathrm{~kb}$, with an estimated 995,540 bp genome size, 844 predicted protein-coding genes, and $25 \%$ GC content. The Nbe genome is circular, 495,352 bp in length, contains 503 protein-coding genes, and has a $22 \%$ GC content. The $\mathrm{N} \beta$ e genome is circular, 866,396 bp in length, contains 880 protein-coding genes, and has a 30\% GC content. Each genome contained hypothetical proteins or uncharacterized proteins making up $14-46 \%$ of predicted protein-coding genes (S Table 1).

\section{Amplicon Diversity}

Amplicon sequencing of Run One resulted in 25,895,690 reads with an average reads per sample of 137,743 . After binning, there were 4,930,010 CO1 reads, 6,491,918 $18 \mathrm{~S}$ reads, and 14,468,228 $16 \mathrm{~S}$ reads. Following assembly in dada2 and decontamination, there were 1,876,107 sequences corresponding to 329 amplicon sequence variants (ASVs) for $18 \mathrm{~S}, 1,522,378$ sequences corresponding to 188 ASVs for C01, and 62,905 sequences with 152 ASVs for 16S. The 18 S data indicated that Nephromyces, like Plasmodium (32), encodes multiple distinct copies of 18S rRNA, making this marker uninformative for species quantification. This maker was subsequently dropped for Run Two and the data are not presented here.

A total of 335 distinct Nephromyces COI ASVs were recovered from samples collected from Molgula manhattensis. Before clustering there was an average of 79.26 ASVs, with a max of 143 ASVs and min of 27 ASVs, per tunicate host (Fig. 3). Clustering at the $98 \%$ and $97 \%$ identity level resulted in an average of 5.1 and 2.8 ASVs per tunicate (Fig. 3). The most common AVSs were found in $53 \%$ of tunicates sampled and the rarest in $2.12 \%$. At the $97 \%$ level, the most common clusters were found in $75.5 \%$ of tunicates the rarest in $2.12 \%$. We identified a total of $15216 \mathrm{~s}$ sequences from the bacterial endosymbionts: 49 from Betaproteobacteria, 89 from 
Alphaproteobacteria, and 14 from Bacteroidetes. The average number of $16 \mathrm{~S}$ sequences per tunicate was 13.94 with a max of 49 and a min of 0 (Fig. 3). Forty percent of the tunicates sampled contained all three types of bacterial endosymbiont, and $90 \%$ contained at least two of the bacterial endosymbiont types. Out of the $50 \mathrm{M}$. manhattensis sampled for amplicons, only two did not contain any 16s endosymbiont sequences, despite containing Nephromyces C01 amplicons.

The 52 Molgula occidentalis samples combined from Runs One and Two had a total of 182 distinct COI ASVs from Nephromyces. Before clustering there was an average of 26.79 ASVs, with a max of 82 and min of 8, per tunicate host (Fig. 3). When clustered to the $97 \%$ identity level there are a total of 19 clusters with an average of 7.8 per tunicate (Fig. 3). The most common AVSs were found in $75 \%$ of tunicates sampled and the rarest ASVs were in 1.9\%. After clustering at $97 \%$, the most common clusters were in $100 \%$ of tunicates the rarest in $1.9 \%$. From a total of 64 16s rRNA sequences classified as bacterial endosymbionts, 24 resolved as Betaproteobacteria, 37 as Alphaproteobacteria, and 13 as Bacteroidetes. The average number of $16 \mathrm{~S}$ sequences per tunicate was 16.7 with a max of 30 and a min of 3 (Fig. 3). Nearly 41\% of samples contained all three types of bacterial endosymbiont, and $80.77 \%$ contained at least two of the endosymbiont types. All $M$. occidentalis collected had at least one bacterial endosymbiont $16 \mathrm{~S}$ sequence.

\section{Molgula manhattensis laboratory culturing}

Regardless of infection status all cultures of $M$. manhattensis reproduced successfully with no apparent differences. Culture with the full Nephromyces inoculum never lost Nephromyces (in one case over 4.5 years, $\sim 9$ generations). An average of 25ng of DNA was extracted from the renal fluid of these tunicates. Cloning Nephromyces COI sequence and the bacterial 16S found this culture had 4 different Nephromyces species two with alphaproteobacteria endosymbionts and two with Bacteroidetes. On the plate inoculated with the 1/100 dilution Nephromyces transmission was also stable and never lost ( 4.5 years, $\sim 9$ generations). DNA extractions of renal fluid from these animals was never detectable by qbit and cloning revealed two Nephromyces species one with alphaproteobacteria and one with betaproteobacteria. Only two cultures were evey successfully inoculated with a single Nephromyces species and Nephromyces was lost from both after two to three generations.. The single-species cell densities were so low that infection status had to be confirmed with PCR amplification as visual inspection with a dissecting microscope often missed the presence of Nephromyces. In both cases the bacterial endosymbiont could not be determined and may not have actually contained a bacterial endosymbiont.

\section{Discussion}

Our combined genomic, microscopy and population data reveals a unique ecosystem within the renal sac of molgulid tunicates, where multiple species of Nephromyces, harboring one of three different strains of bacterial endosymbiont, 
work in concert. The genomes of the three bacterial endosymbiont types are highly reduced, but perform several key functions, as well as complete pathways partially encoded by Nephromyces (Fig. 2). Only $\mathrm{N} \beta$ e is capable of synthesizing a full complement of amino acids (with the exception of tryptophan) with Nephromyces, but FISH microscopy shows only a single endosymbiont type exists within each individual (Fig. 1). Additionally, $\mathrm{N} \beta$ e is missing genes to synthesize essential cofactors, such as lipoic acid, encoded by Nbe and Nae. Together, these data indicate that, not only do individual Nephromyces exchange metabolites with their endosymbiotic bacteria, but between conspecifics within the renal sac.

In support of the importance of multiple species infecting each renal sac, wild tunicates are either infected by multiple species of Nephromyces or have not yet been infected (Fig. 3). In experiments with laboratory raised tunicates, limiting the number of Nephromyces species per host affected cell densities and transmission efficiency. Single-species Nephromyces inoculations in lab raised tunicates grew poorly and were lost in subsequent generations of tunicates. Limited infections with two Nephromyces species were transmitted to other tunicates, but contained an order of magnitude fewer cells (measured by DNA quantities from extractions) than tunicates colonized by four or more species with undiluted renal fluid from wild tunicates.

The multi-species communities Nephromyces forms are a challenge for genomic methods. Nephromyces cells have highly variable morphology (Fig. 1) and rupture rapidly when manually isolated from renal sac fluid. Whereas the transcriptomic data reported here are from a single renal sac, both the genomic and transcriptomic sequences represent a community of closely related Nephromyces. By sequencing multiple species we have recovered the genomes of all three of the bacterial endosymbionts of Nephromyces, providing key insights into how this system functions in vivo.

The estimated 8,000 gene transcriptome for Nephromyces is largely complete as estimated by BUSCO. This number is similar to some of the most gene-rich apicomplexans, such as Toxoplasma gondii, which also encodes 8,000 genes. This is interesting given the phylogenetic placement of Nephromyces in the Hematozoa (19). Hematozoa, which contains the plasmodiidae and piroplasmida lineages, have some of the smallest genomes with the least number of genes of any sequenced apicomplexans. High gene numbers may be reflective of the greater biosynthesis and metabolic capabilities needed for an extracellular lifestyle and horizontal infection via spores in the water column.

Two of the biggest surprises in these datasets both involve purine metabolism. Nephromyces have the metabolic capabilities to convert xanthine into glyoxylate. Glyoxylate can be converted with serine-pyruvate aminotransferase (AGXT) into glycine and pyruvate, and Nephromyces additionally encodes malate synthase (MLS), which combines Glyoxylate and acetyl-CoA into malate. This is proposed to be the primary route of carbon, nitrogen, and energy acquisition for 
Nephromyces (6). This pathway is absent in all available apicomplexans genomes, but it appears the enzymes in this pathway were retained from the last common ancestor of Apicomplexa and not the result of a more recent horizontal gene transfer (6). The second surprise in Nephromyces purine metabolism is de novo purine biosynthesis (Sup Fig 1). While some apicomplexan lineages have one or two genes to synthesize inosine monophosphate (IMP) from immediate precursors, Nephromyces is predicted to encode the entire de novo purine synthesis pathway from 5-Phosphoribosyl diphosphate (PRPP). As the inability to synthesize purines has been widely targeted for drug development against other apicomplexan species, its presence in Nephromyces is unexpected.

The presence of both purine degradation and purine synthesis could be critical to the unusual epidemiology of Nephromyces. Tunicates lack the enzymatic ability to degrade purines past uric acid. By obtaining the bulk of the required carbon, nitrogen, and energy from a tunicate metabolic waste product, Nephromyces is able to limit impact on its host, while still reaching high cellular densities. De novo synthesis of purines means Nephromyces is not dependent on the host for IMP. Purine degradation and purine biosynthesis may have been the critical factors which allowed Nephromyces to leave the intracellular environment and colonize the renal sac.

Another critical factor in the ability of Nephromyces to survive in the renal sac is likely its bacterial endosymbionts. The $\mathrm{N} \alpha$ e found in Nephromyces and Cardiosporidium are monophyletic, indicating that they have been maintained and vertically transmitted since the divergence of Nephromyces and Cardiosporidium (33). In addition to the $\mathrm{N} \alpha$ e, Nephromyces has also acquired a $\mathrm{N} \beta$ e and a $\mathrm{Nbe}$ endosymbiont. The $\mathrm{N} \alpha$ e and Nbe show a marked reduction in carbon metabolism with $\mathrm{N} \alpha$ e only encoding genes for the citric acid cycle, and Nbe only capable of processing three-carbon compounds and encoding a partial citric acid cycle (Fig. 2). Such pronounced reduction suggests that Nephromyces provide their symbionts a limited 'diet' (26). In both symbionts, carbon metabolism may be dependent on pyruvate, which is one of the end products from the degradation of uric acid. All three of the bacterial endosymbionts paradoxically encode complete fatty acid biosynthesis but lack fatty acid degradation (Fig. 2). Presumably, the fatty acid biosynthesis is for the construction of membranes, but without fatty acid degradation, these symbionts are incapable of processing fatty acids as a carbon source. Both $\mathrm{N} \alpha$ e and $\mathrm{N} \beta$ e do not have complete pathways for the creation of glycerophospholipids, yet both contain phospholipid ABC transporters, possibly indicating a dependence on Nephromyces for phospholipids.

None of the three endosymbionts contain any of the genes involved in purine degradation (Fig. 2). Both $\mathrm{N} \beta$ e and $\mathrm{N} \alpha$ e do not encode any genes involved in $d e$ novo purine biosynthesis, including genes for the conversion from IMP to adenine and guanine. Nbe can likely synthesize purines from PPRP though the histidine biosynthesis pathway and contains the genes to synthesize adenine and guanine 
from IMP. The lack of purine biosynthesis genes in both $\mathrm{N} \beta$ e and $\mathrm{N} \alpha$ e makes these symbionts dependent on Nephromyces for both adenine and guanine. If Nephromyces were incapable of de novo purine biosynthesis, then the entire renal sac community would be dependent on either the tunicate host for all purines or on Nbe. This would likely be a significant burden on the host and adds support to the genomic prediction that Nephromyces are able to synthesize purines.

Given their reduced genomes and correspondingly reduced metabolic capabilities, $\mathrm{N} \beta$ e and Nbe encode a large proportion of genes for synthesizing amino acids, vitamins, and co-factors (Fig. 2). Together, $\mathrm{N} \beta$ e and Nbe could provide Nephromyces with all but one essential amino acid (tryptophan). $\mathrm{N} \alpha$ e is only capable of synthesizing three amino acids, and only one which is an essential amino acid for Nephromyces (lysine). Vitamin and cofactor biosynthesis in $\mathrm{N} \alpha$ e is also limited to synthesizing only heme, ubiquinone, and lipoic acid, with lipoic acid being the only product Nephromyces may be incapable of synthesizing itself (Fig. 2). The monophyletic alphaproteobacterium endosymbiont in Cardiosporidium ciona also encodes lipoic acid and was predicted to be an essential function (33). None of three bacterial endosymbiont types are functionally equivalent, and FISH microscopy has not revealed any Nephromyces cell containing more than a single symbiont type.

No wild Molgula manhattensis or Molgula occidentalis we examined contained just a single Nephromyces species. The average population was three to five Nephromyces species in M. manhattensis and seven to eight in the larger $M$. occidentalis, with a minimum of two (Fig. 3). From 50 M. manhattensis collected from a single locality $\sim 21$ species of Nephromyces species were recovered. Results were similar for Molgula occidentalis 60 individuals, 19 Nephromyces species. Whereas single genus multispecies apicomplexan infections are relatively common (34-37), the extreme diversity and universal multispecies infections found in Molgula tunicates is striking. All three bacterial endosymbionts were found in $40 \%$ of M. manhattensis and Molgula occidentalis samples and at least two types were in $90 \%$ of M. manhattensis and $80 \%$ of Molgula occidentalis.

Our data shows that Nephromyces spp. form complex species swarm communities inside the renal sacs of their hosts, where closely related conspecifics and their functionally distinct bacterial endosymbionts are in immediate proximity with each other. Based on the genomic data from the bacterial endosymbionts, no single bacterial endosymbiont is capable of producing all of the metabolites and precursor molecules that are predicted to be essential for Nephromyces. As of yet we have no support of direct metabolite exchange between Nephromyces species, but propose that the production of these metabolites may become available in the renal sac indirectly by leaky membranes or cell lysis. This hypothesis does not preclude that these metabolites may also come from the tunicate host, but it is unlikely the expense of an endosymbiont would be tolerated if these metabolites were available in sufficient quantities. The presence of multiple endosymbionts with different types 
of bacterial endosymbionts may increase the amount and availability of these metabolites inside the renal sac. If this is the case, then multiple Nephromyces species may actually decrease Nephromyces virulence overall consistent with results from (38). In this way, Nephromyces appear to have evolved a complex mutualism with their host, sister species, and bacterial endosymbionts.

\section{Materials \& Methods}

Fluorescence in situ Hybridization (FISH) Microscopy

Three 16S targeted bacterial probes with attached fluorophores were used in each fluorescence in situ hybridization (FISH) reaction. The probes were designed by Seah 2011 to be specific to the classes of bacterial endosymbionts present in Nephromyces. This included alphaproteobacteria (AlexaFluor 555 - 5' GCATACCGCCAGCGTTCGTT 3') , betaproteobacteria (AlexaFluor 647 - 5' GCATCCCGCTAGCGTTCAAT 3'), and bacteroides (AlexaFluor 488 - 5' GCTTGTCGCTAGCGTTTATC 3') (Seah 2011).

Renal sacks from wild Molgula manhattensis collected from Greenwich Bay, Rhode Island, USA, were dissected and emptied onto a positively charged slide. The sample was dried on the slide, and then subjected to a dehydration time series in sterile seawater: $50 \%$ ethanol ( 5 mins), 70\% ethanol ( 8 mins), 95\% ethanol (10 mins), and finally $100 \%$ ethanol (15 mins). A hybridization solution consisting of 0.9M NaCl, 0.02M Tris-Cl (pH 7.4), 0.01\% SDS, 20\% HiDi Formamide, and 5\% dextran sulfate was mixed with $2 \mathrm{pmol} / \mu \mathrm{L}$ of probe, and $200 \mu \mathrm{L}$ applied to the slide (Seah 2011). The slide was then incubated in a humidified dark chamber at 46C overnight. Two washes were conducted by draining the remaining hybridization buffer from the slide and applying $200 \mu \mathrm{L}$ of wash buffer $(0.215 \mathrm{M} \mathrm{NaCl}, 0.02 \mathrm{M}$ Tris-Cl (pH 7.4), 0.01\% SDS) for 30 minutes, with one change of solution (Seah 2011). Samples were then mounted with Prolong Gold w/ DAPI, cured, and imaged using a Zeiss Axioimager M2 imaging system at the URI Genomics and Sequencing Center (URIGSC).

\section{$\underline{\text { RNA Extraction \& Sequencing }}$}

RNA extraction buffer (Zymo Research LLC. Irvine, CA) was added to samples and ground with a pestle. Following grinding, the Zymo Quick-RNA kit (Zymo Research LLC. Irvine, CA) was used and the manufacturer's protocol was followed. RNA was converted to cDNA and sequenced at the School of Medicine Genome Resource Center, University of Maryland. One paired-end RNA library was run on one lane of the Illumina HiSeq platform. Resulting in 40,606,230 reads from the $M$. manhattensis renal sac.

$\underline{\text { RNA Assembly \& Binning }}$ 
Transcriptome data was assembled and proteins were predicted with Trinity/Trinotate pipeline v2.4.0 run on the server at Brown University Center for Computation and Visualization (40). Protein sequences were predicted using Transdecoder (40). BLASTp v 2.8.1 was used to identify bacterial sequences from assembled transcripts against NCBI's refseq database and binned. Orthofinder was run on remaining Eukaryotic sequences with a custom database of alveolate and ascidian transcriptomes.. Including the following apicomplexan and Chromera transcriptomes downloaded from EuPathDB (C. parvum Iowa, G. niphandrodes, $B$. bovis T2Bo, T. parva Muguga, P. falciparum 3D7, C. cayetanensis, E. brunetti Houghton, E. falciformis Bayer Haberkorn, E. tenella Houghton, H. hammondi HH34, N. caninum LIV, S. neurona SN3, T. gondii ME49, C. velia CCMP2878, V. brassicaformis CCMP3155) and ascidian transcriptomes downloaded form aniseed (C. intestinalis, $H$. roretzi, B. schlosseri. M. oculata, $M$. occulta, $M$. occidentalis). Orthologous genes were binned using closest phylogeny in R.

Trimmed reads were mapped back to each of the three bins (Nephromyces, $M$. manhattensis, Nephromyces's bacteria) and then reassembled independently in Spades (41). Nephromyces transcriptome was composed of multiple Nephromyces species CDhit v4.6.8 was used to cluster transcripts based on percent identity (42). Transcriptome completeness was assessed with Busco v3 against the Eukaryotic and bacterial reference data sets (43). Transcripts were annotated using Interproscan v71.0 (44).

\section{Illumina DNA Extraction \& Sequencing}

The renal sacs from 8 lab grown $M$. manhattensis individuals were dissected and their renal fluid was pooled in a $1.5 \mathrm{ml}$ Eppendorf tube. Contents were centrifuged at $8000 \mathrm{~g}$ for $5 \mathrm{~min}$. to pellet Nephromyces cells, and following centrifugation the renal fluid was discarded. Five hundred microliters of CTAB buffer with $5 \mathrm{ul}$ of proteinase $\mathrm{K}$ and ceramic beads were added to the pelleted Nephromyces cells. The sample was placed in a bead beater for $3 \mathrm{~min}$. and then on a rotator for $1.5 \mathrm{hrs}$ at room temp. Five hundred microliters of chloroform were added, mixed gently and centrifuged for $5 \mathrm{~min}$. The top layer was removed and $2 \mathrm{x}$ the sample volume of ice-cold $100 \%$ EtOH and $10 \%$ sample volume of $3 \mathrm{M}$ sodium acetate were added to the sample and incubated a -20C overnight. The sample was centrifuged at $16000 \mathrm{xg}$ for $30 \mathrm{~min}$. and the liquid was removed. Ice cold $70 \% \mathrm{EtOH}$ was added and centrifuged at $16000 \mathrm{xg}$ for $15 \mathrm{~min}$. The liquid was removed and the sample air-dried for 2 min. DNA was re-eluted in 50ul of deionized water. A nanodrop (2000c, ThermoScientific) was used to assess DNA purity and DNA concentration, and a genomic gel was run to assess DNA fragmentation. Following quality control, an Illumina library was constructed. Library prep and sequencing were done at the URI Genomics and Sequencing Center (URIGSC). The completed library was sequenced on the Illumina Miseq platform at the URIGSC and the HiSeq platform at the University of Maryland Baltimore sequencing center on three lanes. 


\section{Illumina Assembly}

One MiSeq lane and three lanes of HiSeq, all from the same library, were trimmed using Trimmomatic v0.39 (45) then assembled using SPAdes v3.13.0 (41) assembler on the URI server BlueWaves.

\section{Pacific Biosciences DNA Extraction \& Sequencing}

Using the contents of 150 (done in batches of 10 then pooled) M. manhattensis renal sacs, the same DNA extraction protocol was performed as for Illumina sequencing. DNA was sequenced using three SMRT cells on the Pacific Biosciences platform at the University of Baltimore sequencing center.

\section{$\underline{\text { Pacific Biosciences Assembly }}$}

Pacific Biosciences reads were error corrected using PBSuite v15.8.24 (46) on the Brown University server, Oscar. Reads were then assembled using Canu v1.6 (47). Contigs generated by Canu were combined with Illumina MiSeq/HiSeq short reads with ABySS v2.02 (48). Nephromyces contigs were identified by mapping Nephromyces transcriptome reads to the genomic assembly using Bowtie2 v1.2.2. Contigs with greater than 90x coverage assessed by bedtools (49) were binned as Nephromyces additional screening of binned contigs was done using VizBin (50). Contigs were additionally binned using CAT (51), and those that were classified as apicomplexan were added to the existing assembly.

\section{Bacterial Endosymbiont Genome Assembly}

Using the contigs from the ABySS assembly bacterial contigs were initially identified by hexamers using VizBin, transcriptomic reads that were identified as bacterial were mapped using Bowtie2 (52). Bacterial contigs were separated based on a 90x coverage threshold with bedtools (49). Binned bacterial contigs were preliminarily annotated with Prokka (53). Resulting annotations were run through KEGG GhostKoala to assign and separate by taxonomy. Taxon separated contig bins were merged and scaffolded using PBJelly from the PBsuite v15.8.24 of tools (54). Illumina MiSeq and HiSeq reads were remapped to resulting contigs to ensure accurate assembly using Bowtie2. Final assembled bacterial genomes were re-annotated with Prokka using a genus-specific database.

Data

The pangenome and transcriptome of Nephromyces, as well the associated bacterial endosymbionts, are available on GenBank under the bioproject ID PRJNA666913.

\section{Abridged Amplicon Methods}


Fifty Molgula manhattensis tunicates were collected from a single floating dock located in Greenwich Bay, RI (41.653N, -71.452W), and 54 Molgula occidentalis were collected from Alligator Harbor, FL (29.899N, -84.381W) by Gulf Specimens Marine Laboratories, Inc. (https://gulfspecimen.org/). DNA was extracted with previously described methods. Nephromyces specific COI primers and universal $16 \mathrm{~S}$ rRNA primers (55) were PCR amplified, and sequenced on two Illumina Miseq runs (2x250 2x200). Resulting sequencing reads were error corrected and merged using dada2 with pool=sudo (56). Amplicon sequence variants (ASVs) were stringently filtered to only ASVs with greater than 20 reads per sample. Nephromyces COI ASVs were clustered based on percent identity from 100-94\% with CD-hit. For full methods see supplemental materials.

\section{Molgula manhattensis laboratory culture}

Molgula manhattensis tunicates were collected from a dock in Greenwich Bay, Rhode Island (41.653N, -71.452W) during the summer of 2014. In batches of five Gonads were dissected from sexually mature, M. manhattensis. Eggs and sperm were mixed with sterile seawater and divided evenly between two petri dishes. This was repeated 4 times for a total of eight plates. Plates were incubated at room temperature for two days with daily $100 \%$ water changes. Tunicate larvae attached to the bottom and sides of the petri dishes by day three. By day four, larvae had metamorphosed into adults and were actively feeding. Plates were moved to an incubator at $18^{\circ} \mathrm{C}$ with a $24 \mathrm{hr}$ dark cycle to limit growth of contaminants. Tunicates were fed by $100 \%$ water exchange with cultures of Isochrysis galbana and Chaetoceros gracilis three days a week. After ten days a single M. manhattensis was dissected and the renal fluid was extracted with a syringe and placed in a $1.5 \mathrm{ml}$ tube. $1 \mu$ or renal fluid was added to two plates, $1 \mu$ of renal fluid diluted $1 / 100$ was added to two plates, and a single oocyst was picked and added to two plates, and two plates were kept Nephromyces free. After several weeks tunicates were moved to aerated beakers to meet their increased nutrient and gas exchange requirements. Feeding regimen remained the same except that food volume was increased with tunicate growth. Tunicates were grown for six months until they were $\sim 10 \mathrm{~mm}$ across. After 6 months tunicates were periodically sacrificed to cheek infection status 
1. C. M. Cavanaugh, S. L. Gardiner, M. L. Jones, H. W. Jannasch, J. B. Waterbury, Prokaryotic Cells in the Hydrothermal Vent Tube Worm Riftia pachyptila Jones: Possible Chemoautotrophic Symbionts. Science 213, 340-342 (1981).

2. A. Orsi, et al., Dynamic and transient interactions of Atg9 with autophagosomes, but not membrane integration, are required for autophagy. Mol. Biol. Cell 23, 1860-1873 (2012).

3. M. B. Saffo, Nitrogen Waste or Nitrogen Source? Urate Degradation in the Renal Sac of Molgulid Tunicates. Biol. Bull. 175, 403-409 (1988).

4. M. B. Saffo, Symbiosis within a symbiosis: Intracellular bacteria within the endosymbiotic protistNephromyces. Mar. Biol. 107, 291-296 (1990).

5. M. B. Saffo, A. M. McCoy, C. Rieken, C. H. Slamovits, Nephromyces, a beneficial apicomplexan symbiont in marine animals. Proc. Natl. Acad. Sci. 107, 16190-16195 (2010).

6. C. Paight, C. H. Slamovits, M. B. Saffo, C. E. Lane, Nephromyces Encodes a Urate Metabolism Pathway and Predicted Peroxisomes, Demonstrating That These Are Not Ancient Losses of Apicomplexans. Genome Biol. Evol. 11, 41-53 (2019).

7. A. A. Escalante, F. J. Ayala, Evolutionary origin of Plasmodium and other Apicomplexa based on rRNA genes. Proc. Natl. Acad. Sci. 92, 5793-5797 (1995).

8. N. A. Counihan, M. Kalanon, R. L. Coppel, T. F. de Koning-Ward, Plasmodium rhoptry proteins: why order is important. Trends Parasitol. 29, 228-236 (2013).

9. R. Cardoso, H. Soares, A. Hemphill, A. Leitão, Apicomplexans pulling the strings: manipulation of the host cell cytoskeleton dynamics. Parasitology 143, 957-970 (2016).

10. A. J. Reid, Large, rapidly evolving gene families are at the forefront of host-parasite interactions in Apicomplexa. Parasitology 142, S57-S70 (2015).

11. J. Janouškovec, et al., Factors mediating plastid dependency and the origins of parasitism in apicomplexans and their close relatives. Proc. Natl. Acad. Sci. 112, 10200-10207 (2015).

12. Y. H. Woo, et al., Chromerid genomes reveal the evolutionary path from photosynthetic algae to obligate intracellular parasites. eLife 4 (2015).

13. M. Zarowiecki, M. Berriman, What helminth genomes have taught us about parasite evolution. Parasitology 142, S85-S97 (2015).

14. S. A. Frank, Host-Symbiont Conflict over the Mixing of Symbiotic Lineages. Proc. Biol. Sci. 263, 339-344 (1996).

15. S. Alizon, A. Hurford, N. Mideo, M. Van Baalen, Virulence evolution and the trade-off hypothesis: history, current state of affairs and the future: Virulence evolution and trade-off hypothesis. J. Evol. Biol. 22, 245-259 (2009).

16. C. E. Cressler, D. V. McLEOD, C. Rozins, J. Van Den Hoogen, T. Day, The adaptive evolution of virulence: a review of theoretical predictions and empirical tests. Parasitology 143, 915-930 (2016).

17. S. A. Frank, Models of Parasite Virulence. Q. Rev. Biol. 71, 37-78 (1996).

18. M. B. Saffo, R. Nelson, The cells of Nephromyces : developmental stages of a single life cycle. Can. J. Bot. 61, 3230-3239 (1983).

19. S. A. Muñoz-Gómez, et al., Nephromyces Represents a Diverse and Novel 
Lineage of the Apicomplexa That Has Retained Apicoplasts. Genome Biol. Evol. 11, 2727-2740 (2019).

20. J. R. Nolfi, Biosynthesis of uric acid in the tunicate, Molgula manhattensis, with a general scheme for the function of stored purines in animals. Comp. Biochem. Physiol. 35, 827-842 (1970).

21. C. C. Lambert, G. Lambert, G. Crundwell, K. Kantardjieff, Uric Acid Accumulation in the Solitary Ascidian. 10 (1998).

22. M. A. Rouf, R. F. Lomprey, Degradation of Uric Acid by Certain Aerobic Bacteria. J. Bacteriol. 96, 617-622 (1968).

23. A. Thong-On, et al., Isolation and Characterization of Anaerobic Bacteria for Symbiotic Recycling of Uric Acid Nitrogen in the Gut of Various Termites. Microbes Environ. 27, 186-192 (2012).

24. W. J. Middelhoven, G. S. De Hoog, S. Notermans, Carbon assimilation and extracellular antigens of some yeast-like fungi. Antonie Van Leeuwenhoek 55, 165-175 (1989).

25. J. F. Yamagishi, N. Saito, K. Kaneko, The advantage of leakage of essential metabolites and resultant symbiosis of diverse species. Phys. Rev. Lett. 124, 048101 (2020).

26. N. A. Moran, H. E. Dunbar, J. L. Wilcox, Regulation of Transcription in a Reduced Bacterial Genome: Nutrient-Provisioning Genes of the Obligate Symbiont Buchnera aphidicola. J. Bacteriol. 187, 4229-4237 (2005).

27. M. J. López-Sánchez, et al., Evolutionary Convergence and Nitrogen Metabolism in Blattabacterium strain Bge, Primary Endosymbiont of the Cockroach Blattella germanica. PLoS Genet. 5, e1000721 (2009).

28. D. J. de Souza, A. Bézier, D. Depoix, J.-M. Drezen, A. Lenoir, Blochmannia endosymbionts improve colony growth and immune defence in the ant Camponotus fellah. BMC Microbiol. 9, 29 (2009).

29. H. Urakawa, et al., Hydrothermal vent gastropods from the same family (Provannidae) harbour e- and gamma-proteobacterial endosymbionts. Environ. Microbiol. 7, 750-754 (2005).

30. B. Marin, E. C. M. Nowack, M. Melkonian, A Plastid in the Making: Evidence for a Second Primary Endosymbiosis. Protist 156, 425-432 (2005).

31. B. Seah, "A Tripartite Animal-Protist-Bacteria Symbiosis: Culture-Independent and Phylogenetic Characterization." (2011).

32. Y. Nishimoto, et al., Evolution and phylogeny of the heterogeneous cytosolic SSU rRNA genes in the genus Plasmodium. Mol. Phylogenet. Evol. 47, 45-53 (2008).

33. Elizabeth S. Hunter, Christopher Paight, Christopher E. Lane, Metabolic contributions of an alphaproteobacterial endosymbiont in the apicomplexan Cardiosporidium cionae. Front. Microbiol. (2020).

34. T. J. C. Anderson, et al., Microsatellite Markers Reveal a Spectrum of Population Structures in the Malaria Parasite Plasmodium falciparum. Mol. Biol. Evol. 17, 1467-1482 (2000).

35. K.-S. Lee, et al., Plasmodium knowlesi: Reservoir Hosts and Tracking the Emergence in Humans and Macaques. PLoS Pathog. 7, e1002015 (2011). 
36. A. Arnott, A. E. Barry, J. C. Reeder, Understanding the population genetics of Plasmodium vivax is essential for malaria control and elimination. Malar. J. 11, 14 (2012).

37. A. Lalremruata, et al., Species and genotype diversity of Plasmodium in malaria patients from Gabon analysed by next generation sequencing. Malar. J. 16 (2017).

38. P. Nelson, G. May, Defensive Symbiosis and the Evolution of Virulence. Am. Nat. 196, 333-343 (2020).

39. Y.-L. Lau, et al., Deciphering the Draft Genome of Toxoplasma gondii RH Strain. PLoS ONE 11 (2016).

40. B. J. Haas, et al., De novo transcript sequence reconstruction from RNA-seq using the Trinity platform for reference generation and analysis. Nat. Protoc. 8, 1494-1512 (2013).

41. A. Bankevich, et al., SPAdes: A New Genome Assembly Algorithm and Its Applications to Single-Cell Sequencing. J. Comput. Biol. 19, 455-477 (2012).

42. W. Li, A. Godzik, Cd-hit: a fast program for clustering and comparing large sets of protein or nucleotide sequences. Bioinformatics 22, 1658-1659 (2006).

43. F. A. Simão, R. M. Waterhouse, P. Ioannidis, E. V. Kriventseva, E. M. Zdobnov, BUSCO: assessing genome assembly and annotation completeness with single-copy orthologs. Bioinformatics 31, 3210-3212 (2015).

44. R. D. Finn, et al., InterPro in 2017-beyond protein family and domain annotations. Nucleic Acids Res. 45, D190-D199 (2017).

45. A. M. Bolger, M. Lohse, B. Usadel, Trimmomatic: a flexible trimmer for Illumina sequence data. Bioinformatics 30, 2114-2120 (2014).

46. A. C. English, et al., Mind the Gap: Upgrading Genomes with Pacific Biosciences RS Long-Read Sequencing Technology. PLoS ONE 7, e47768 (2012).

47. S. Koren, et al., Canu: scalable and accurate long-read assembly via adaptive $k$ -mer weighting and repeat separation. Genome Res. 27, 722-736 (2017).

48. S. D. Jackman, et al., ABySS 2.0: resource-efficient assembly of large genomes using a Bloom filter. Genome Res. 27, 768-777 (2017).

49. A. R. Quinlan, I. M. Hall, BEDTools: a flexible suite of utilities for comparing genomic features. Bioinformatics 26, 841-842 (2010).

50. C. C. Laczny, et al., VizBin - an application for reference-independent visualization and human-augmented binning of metagenomic data. Microbiome 3, 1 (2015).

51. F. A. B. von Meijenfeldt, K. Arkhipova, D. D. Cambuy, F. H. Coutinho, B. E. Dutilh, Robust taxonomic classification of uncharted microbial sequences and bins with CAT and BAT. bioRxiv (2019) https:/doi.org/10.1101/530188 (April 4, 2019).

52. B. Langmead, S. L. Salzberg, Fast gapped-read alignment with Bowtie 2. Nat. Methods 9, 357-359 (2012).

53. T. Seemann, Prokka: rapid prokaryotic genome annotation. Bioinformatics 30, 2068-2069 (2014).

54. B. Bushell, BBMap. SourceForge (2014) (August 11, 2020).

55. A. Klindworth, et al., Evaluation of general $16 \mathrm{~S}$ ribosomal RNA gene PCR 
primers for classical and next-generation sequencing-based diversity studies. Nucleic Acids Res. 41, e1-e1 (2013).

56. B. J. Callahan, et al., DADA2: High resolution sample inference from Illumina amplicon data. Nat. Methods 13, 581-583 (2016).

57. M. D. Lee, GToTree: a user-friendly workflow for phylogenomics. Bioinformatics 35, 4162-4164 (2019).

58. S. R. Eddy, Accelerated Profile HMM Searches. PLoS Comput. Biol. 7, e1002195 (2011).

59. S. Capella-Gutiérrez, J. M. Silla-Martínez, T. Gabaldón, trimAl: a tool for automated alignment trimming in large-scale phylogenetic analyses. Bioinformatics 25, 1972-1973 (2009).

60. D. Hyatt, et al., Prodigal: prokaryotic gene recognition and translation initiation site identification. BMC Bioinformatics 11, 119 (2010).

61. R. C. Edgar, MUSCLE: a multiple sequence alignment method with reduced time and space complexity. BMC Bioinformatics 5, 113 (2004).

62. W. Shen, J. Xiong, "TaxonKit: a cross-platform and efficient NCBI taxonomy toolkit" (Bioinformatics, 2019) https:/doi.org/10.1101/513523 (October 7, 2020).

63. M. N. Price, P. S. Dehal, A. P. Arkin, FastTree 2 - Approximately Maximum-Likelihood Trees for Large Alignments. PLoS ONE 5, e9490 (2010).

64. G. M. Boratyn, J. Thierry-Mieg, D. Thierry-Mieg, B. Busby, T. L. Madden, Magic-BLAST, an accurate DNA and RNA-seq aligner for long and short reads. bioRxiv (2018) https:/doi.org/10.1101/390013 (February 24, 2019).

65. M. Martin, Cutadapt removes adapter sequences from high-throughput sequencing reads. EMBnet.journal 17, 10-12 (2011).

66. L. Guillou, et al., The Protist Ribosomal Reference database (PR2): a catalog of unicellular eukaryote Small Sub-Unit rRNA sequences with curated taxonomy. Nucleic Acids Res. 41, D597-D604 (2012).

67. K. Katoh, D. M. Standley, MAFFT Multiple Sequence Alignment Software Version 7: Improvements in Performance and Usability. Mol. Biol. Evol. 30, 772-780 (2013).

68. H. Wickham, Create Elegant Data Visualisations Using the Grammar of Graphics (2016) (September 3, 2020).

69. S. J. Booker, Unraveling the Pathway of Lipoic Acid Biosynthesis. Chem. Biol. 11, 10-12 (2004). 


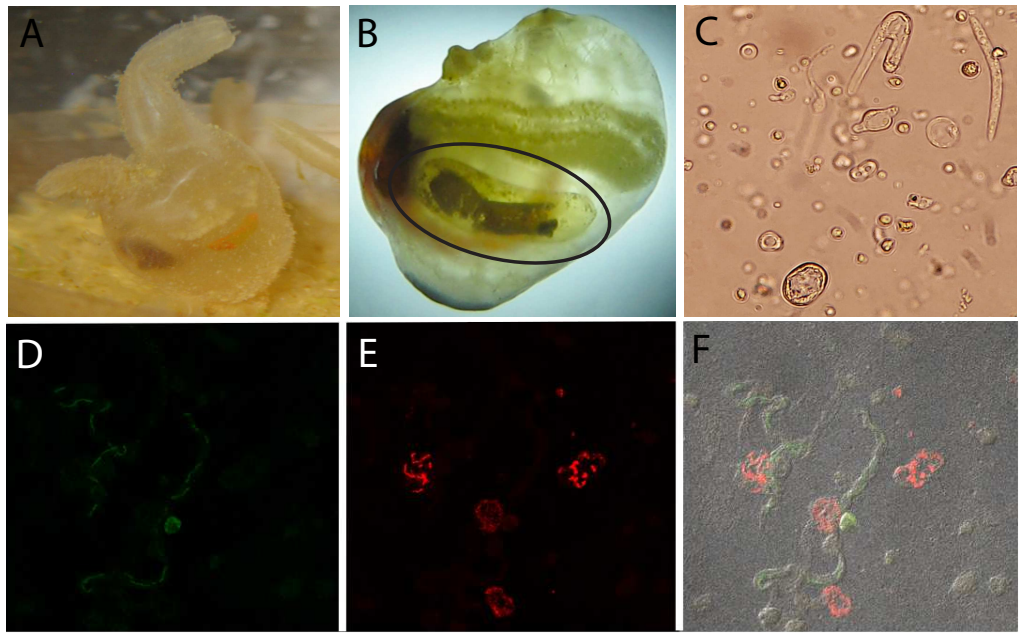


A.

B.
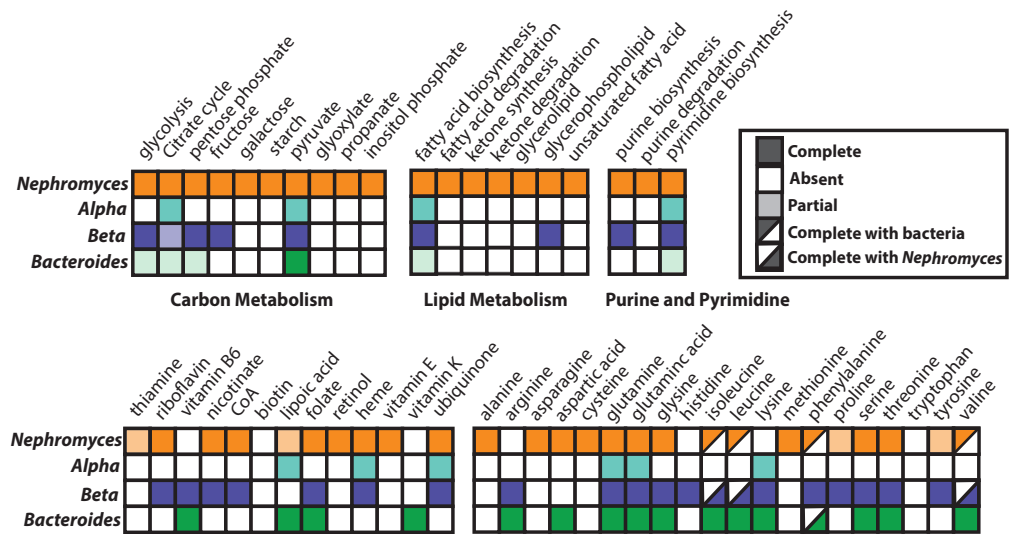

Vitamin and Co-factor Biosynthesis

Amino Acid Biosynthesis 
bioRxiv preprint doi: https://doi.org/10.1101/2020.10.18.344572; this version posted October 18, 2020. The copyright holder for this preprint (which was not certified by peer review) is the author/funder, who has granted bioRxiv a license to display the preprint in perpetuity. It is made

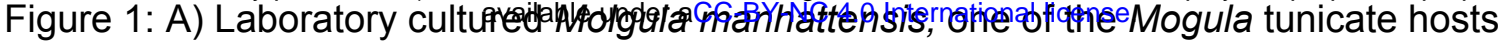

examined in this study. B) Molgula manhattensis with tunic removed to show renal sac (circled), where Nephromyces spp. spend their entire life cycle. The brown substance inside the renals sac are concretions of crystallized uric acid and calcium oxalate. C) Light microscopy photo of several Nephromyces life stages, all cells in photo are of Nephromyces. Bacteiodetes (D) and alphaprotebacterial (E) endosymbionts labeled with $16 S$ rRNA class specific probes, using fluorescence in situ hybridization. F) The composite image of (D) and (E) showing these endosymbionts localized to different Nephromyces cells.

Figure 2: Graphical representation of metabolic pathways in Nephromyces and the three types of bacterial endosymbionts. Filled boxes represent complete pathways, empty boxes indicate no genes in the pathway are encoded, light shading represent partially complete pathways, and half filled boxes indicate pathways that require genes encoded by Nephromyces and a bacterial endosymbiont. A) Carbon, lipid and nucleotide metabolism showing greatly reduced capabilities of the bacterial endosymbionts. B) Amino acid, vitamin, and cofactor metabolism showing strong complementary biosynthetic integration of the bacterial endosymbionts and Nephromyces.

Figure 3: Biodiversity data across Nephromyces and their bacterial endosymbionts. A) Number of Nephromyces ASVs per tunicate individual (blue $=M$. occidentalis, red $=M$. manhattensis). The $\mathrm{x}$-axis shows the different percent identity levels that the ASVs were clustered at. The mean at every clustering level for each host species is shown. B) The number and type of bacterial endosymbiont found from each tunicate host. Bacterial ASV's were not clustered by percent identity. The mean for each host is shown. 